\title{
A Rare Case of Unilateral Bartholin's Gland Cyst in a Murrah Crossbred Buffalo
}

\author{
Sanjay Kumar*
}

Surgical Specialist, Veterinary Polyclinic, Sangrur, Punjab, India

*Corresponding author:

\section{A B S T R A C T}

\section{Keywords}

Bartholin gland Cyst, Murrah buffalo,

Vaginal lubrication, Cyst

Article Info

Accepted:

20 November 2018

Available Online:

10 December 2018
The Bartholin's glands or greater vestibular glands are two glands located slightly posterior and to the left and right of the opening of the vaginal wall about $2.5 \mathrm{~cm}$ caudal to vagina. A 6 years old Murrah cross bred buffalo was reported as vaginal prolapse. Clinical examination revels, is unilateral bartholin gland cyst having $6 \times 5.5 \mathrm{~cm}$ diameter. It was treated with $2 \%$ Lugol's iodine solution, after evacuation of fluid from the cyst under caudal epidural anesthesia. After treatment animal recovered uneventfully and there is no recurrence. This is the first case report in buffalo.

\section{Introduction}

In 1677, a Dutch anatomist Casper Bartholin first described the Bartholin glands. Bartholin Glands are paired and are found in the labia minora of vagina which secrete mucus most actively at the time of estrus. These glands normally open to lateral wall of vestibule about $2.5 \mathrm{~cm}$ caudal to the vagina by a single duct (Roberts, 1971). Normally, they are nonpalpable. Their function is to maintain the moisture of the vaginal mucosa's vestibular surface.

\section{Case report}

A 6 years old Murrah cross bred buffalo was presented with the complaint stating that the vaginal prolapsed while animal resting and slight straining while urinating since 5-6 months. Upon Clinical examination of vagina, a round enlargement covered with vaginal mucosa protruding between the vulvar lips (Fig. 1 and 2) was noticed. The thin walled fluctuating cyst about $6 \times 5.5 \mathrm{~cm}$ in diameter covered with vaginal mucosa and protruding on the right lateral wall of the vagina which was not showed pain on palpation. All other vital parameters were within the normal range.

\section{Treatment and Discussion}

The area around cyst was washed with light $\mathrm{KMnO}_{4}$ Solution. Caudal epidural anesthesia with Inj. Lignocanine 2\% @ $1 \mathrm{ml}$ per $100 \mathrm{~kg}$ 
B.Wt, induces to operate the cyst. Exploratory puncture done with $18 \mathrm{G}$ needle revealed the presence of thick milky fluid in the cyst. The volume of the fluid was approximately $30 \mathrm{ml}$. Then $20 \mathrm{ml}$ of $2 \%$ of Lugol's iodine solution was infused into the cavity following evacuation of the contents of the bartholin gland cyst under caudal epidural anesthesia. Followed by injection Meloxicam (15ml - Intramuscular) and Inj. Chlorphenaramine maleate $(10 \mathrm{ml}$ Intramuscular) for 3 days and advised to feed Mineral Mixture (Pulv. MVM- A Product of Zenegal Neutraceuticals). After 3 Days the buffalo was examined again, nothing was found abnormal at that site of cyst. The Buffalo was recovered uneventfully.
Initially, the bartholin gland cyst was mistaken for vaginal prolapse and the condition was diagnosed as Unilateral Bartholin cyst. Fathalla et al., (1978) mentioned that trauma and extension at the time of calving leads to local vulvo-vaginitis.

In the present study, the bartholin gland cyst reported unilaterally in young Murrah cross bred buffalo. This is not in accordance with Roberts (1971) who stated that it occurs mostly in old age animals. Selvaraju et al., (2010), Manokaran et al., (2014) and Soundararajan et al., (2015) also reported unilateral Bartholin gland cyst in aged Jersey, Holstein Frisian (HF) and Jersey Crossbred cow respectively.

Fig.1\&2 Bartholin Gland cyst in buffalo and Close View of Bartholin Gland Cyst in Buffalo

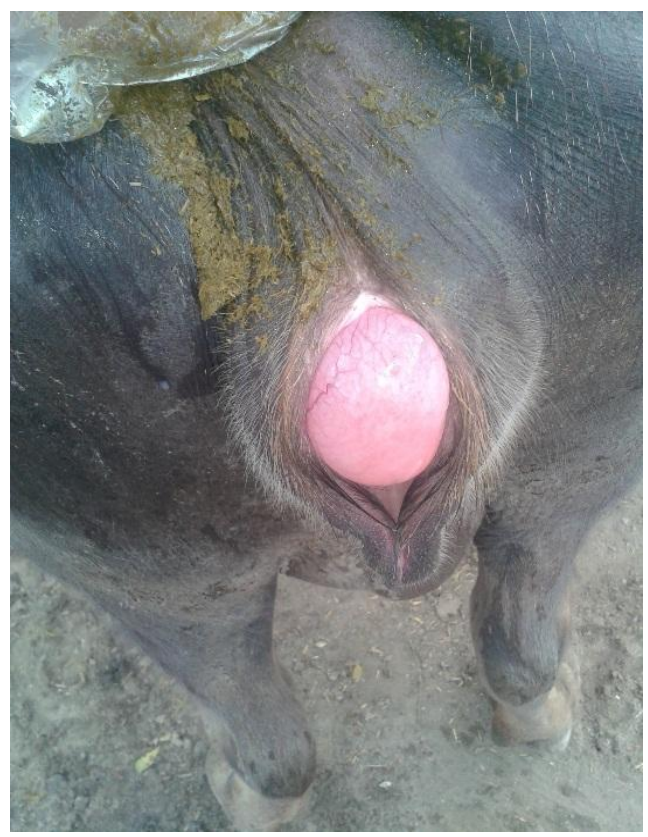

This is in accordance with Bademkiran et al., (2009) who reported Bartholin's gland cyst in $\mathrm{HF}$ heifer. Hence, this is the first report in buffalo.

The cyst causes no problems at parturition nor interferes with conception as stated by Roberts (1971). However sometime, it

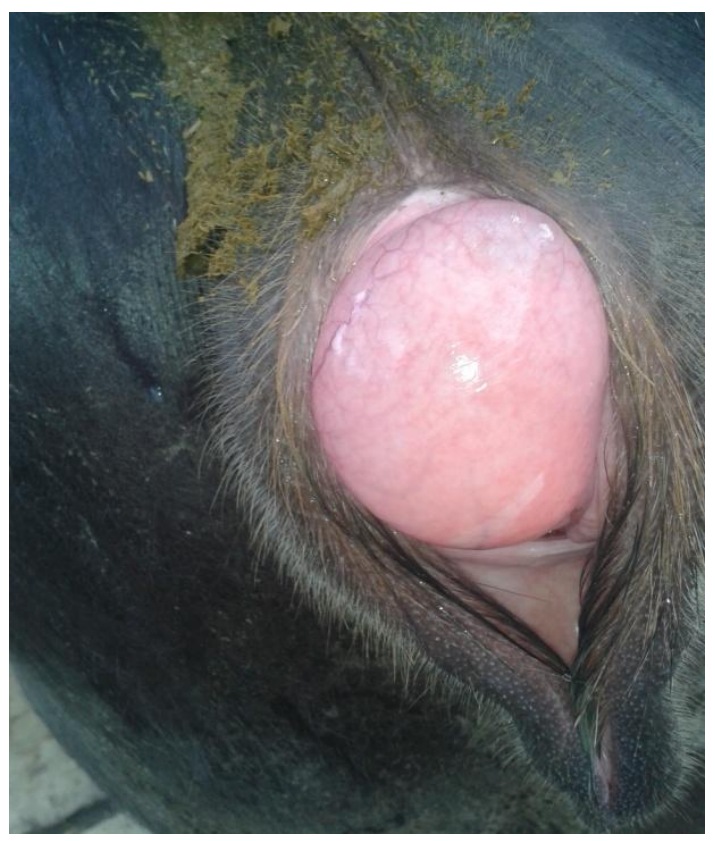

interferes during Artificial Insemination (AI) while inserting AI gun through vagina. The vulvar lips are kept open by the cystic enlargement leads to misunderstanding by owner for prolapse, contamination of the vagina, vestibule and vulva with feces may occur. The shape of the external genitalia was distorted and a degree of vaginitis was noted 
associated with this defect if not treated early. McEntee (1970) considered the presence of Bartholin glands as reservoirs for pathogenic organism. Brucella abortus and B. melitenis were also isolated in some cases of abscess of Bartholin's gland (Fathella et al., 1978 and Peled et al., 2004). Formation of retention cysts arising from occlusive lesions of the duct, as in metaplasia of the lining epithelium associated with chronic hyper-estrogenism in cows with ovarian-follicular cysts.

\section{References}

Fathalla, M., M.S.S. Abdou and H. Fahmi (1978). Bartholin Gland Cyst in Cow. Can. vet. J. 19: 340.

Manokaran, S., Sivasankar, K., Palansamy, M., Selvaraju, M. and Ezakial Napolean, R. (2014). Unilateral Bartholin's gland cyst in a Holstein Friesian crossbred cow. Int. J. Livest. Res., 4(9): 48-50.
McEnteek, K. (1970). The female genital system - In Pathology of Domestic Animals. Jubb and Kennedy, New York and London: Academic Press, 1; pp 487-585.

Peled, N., David, Y. and Yagupsky, P. (2004). Bartholin's gland abscess caused by Brucella melitensis. J. Clinical Microbio., 42 (2): 917-918.

Roberts, S. J. (1971). Veterinary Obstetrics and Genital Diseases. Published by the author. Ithaca, N.Y. Pp, 496.

Selvaraju, M., Palanisamy, M., Prabaharan, V., Ravikumar, K., Ravi, R. and Chandrahasan, C. (2010). Cystic Bartholin's gland in a Jersey crossbred cow: A case report. Indian J. Field. Vet.5(4): 74.

Soundararajan, C., M. Arul Parkesh and K. Senthil Kumar (2015). Unilateral Bartholin gland cyst in a jersey crossbred cow. Ind. J. Ani. Repro. 36(2). Pp 75-76.

\section{How to cite this article:}

Sanjay Kumar. 2018. A Rare Case of Unilateral Bartholin's Gland Cyst in a Murrah Crossbred Buffalo. Int.J.Curr.Microbiol.App.Sci. 7(12): 2851-2853.

doi: https://doi.org/10.20546/ijcmas.2018.712.324 